\title{
Comparação do uso do QFD no Brasil e no Reino Unido
}

\author{
José Antonio Carnevalli \\ Programa de Pós Graduação em Engenharia de Produção (FEMP), UNIMEP \\ Andreza Sassi \\ Faculdade de Engenharia e Ciências Químicas (FECQ), UNIMEP \\ Paulo A. Cauchick Miguel \\ Professor Titular da Faculdade de Eng $\underline{\underline{a}}$ Mecânica e de Produção da UNIMEP \\ Núcleo de Gestão da Qualidade \& Metrologia, Faculdade de Engenharia Mecânica e de Produção, Universidade \\ Metodista de Piracicaba (UNIMEP)
}

O QFD é um método de desenvolvimento de produtos e serviços que vem sendo usado em vários países do mundo. Procurando verificar quais são os verdadeiros benefícios e dificuldades de se implementar este método, alguns pesquisadores tem realizado estudos tipo survey em seus países. Este artigo apresentará uma comparação de duas destas pesquisas, uma realizada no Reino Unido e outra realizada no Brasil. O objetivo desta comparação é verificar quais são os aspectos da aplicação do QFD comuns nos dois países.

Palavras chave: desdobramento da função qualidade; QFD; planejamento da qualidade.

QFD is a method for developing products and services which has been used in many countries the world. For order to verify benefits and difficulties to implement the method, some researchers have realized surveys in their respective countries. This paper shows a comparison of the two research, conducted in the UK and another one performed in Brazil. The objective of this comparison is to verify which aspects of QFD application are common in the two countries.

Keywords: quality function deployment; QFD; quality planning.

\section{Introdução}

O Desdobramento da Função Qualidade (QFD) é um método japonês, hoje aplicado em praticamente todo o mundo, para desenvolvimento de produto e serviço do tipo solução antecipada de problemas. Segundo Akao (1996), o QFD busca resolver as falhas de desenvolvimento ainda na fase de projeto, diferentemente do modelo de solução de problemas após o início da produção, o que gera grandes despesas.

O QFD tem os seguintes objetivos (Cheng et al., 1995): garantir a qualidade desde a fase de projeto, identificando e traduzindo os requisitos dos clientes em características técnicas, desenvolvendo assim, produtos e serviços que os satisfaçam.

A partir da década de 80, o QFD começou a ser utilizado em outros países, como EUA, Reino Unido, Suécia, Brasil, dentre outros. Com o objetivo de verificar quais são os benefícios e dificuldades de se implementar o QFD, alguns pesquisadores realizaram estudos tipo survey em seus países. Este trabalho apresenta a comparação de duas destas pesquisas, uma realizada no Reino Unido em 1999 e outra realizada no Brasil em 2001. Como esses estudos utilizaram metodologias de pesquisa muito semelhantes, tinham o mesmo objetivo e encontraram um número parecidos de empresas utilizando o QFD, pretende-se verificar quais são os aspectos da aplicação do QFD que são similares nos dois países.

\section{Referencial teórico}

Serão apresentados, a seguir, alguns dos conceitos sobre o QFD e exemplos de suas aplicações.

\subsection{Conceito do QFD}

O QFD é um método que ouve os desejos, requisitos e exigências dos clientes, transformando estes dados em características mensuráveis de um produto ou 
serviço. Deste modo, a empresa toma consciência de quais são as características mais importantes para os seus clientes, que devem ser priorizadas no projeto para satisfazê-los.

Existem várias versões do QFD, entre elas o QFD das Quatro Ênfases desenvolvida por Akao e Mizuno (Akao, 1996; Cheng et al., 1995). Esta versão é formada por duas partes: o Desdobramento da Qualidade (QD) e pelo Desdobramento da Função Qualidade no sentido restrito (QFDr). O QD envolve uma série de matrizes, desdobrando as exigências dos clientes em características da qualidade e estas vão sendo desdobradas em funções do produto, subsistemas, componentes, processo e matéria-prima, considerando-se a qualidade, a tecnologia, os custos e a confiabilidade de forma a assegurar a qualidade (Cheng et al., 1995). O QFDr é o desdobramento detalhado das funções de trabalhos necessários para atingir a qualidade (Ohfuji et al., 1997). O QD estabelece o valor dos parâmetros de controle dos processos e completa, junto com QFDr, o padrão de processo. Além disso, como resultado do QFDr, são definidos o Padrão Gerencial do Desenvolvimento de Produto e o Plano de Atividade do Desenvolvimento do Produto (Cheng et al., 1995).

Segundo Cheng et al. (1995) as outras versões do QFD aplicadas nos EUA e na Europa levam em conta apenas o QD, não atingindo assim todos os benefícios do QFD amplo. Cabe então à cada empresa escolher qual é a versão mais adequada a ser aplicada.

Entre os principais benefícios sobre a utilização do QFD, encontradas na literatura, pode-se citar: diminuição no número de modificações de projetos; diminuição do ciclo de desenvolvimento do produto; facilidade de definição da Qualidade Projetada e identificação dos gargalos de engenharia (Ohfuji et al., 1997); aumento da satisfação dos clientes e diminuição do número de reclamações; melhoria da comunicação entre os departamentos; redução de custos no início da produção do novo produto (Cheng et al., 1995).

\subsection{Aplicação do QFD}

O QFD pode ser utilizado para desenvolver produtos e serviços. A seguir, alguns exemplos da sua aplicação.

O método do QFD foi utilizado na GM, inicialmente, para desenvolver componentes como o desembaçador de pará-brisa, sendo mais tarde usado para desenvolver o produto completo. Para reduzir o tamanho das matrizes, a GM criou o "Processo de QFD Simplificado” (The Streamlined QFD Process). Segundo Ross (1999), por este processo, após a empresa definir os requisitos dos clientes, ela faz uma análise identificando os requisitos mais importantes para fazer parte da matriz. Do mesmo modo, ao se desdobrar as características da qualidade, somente as mais importantes farão parte da matriz. Deste modo, a GM conseguiu não somente reduzir o tamanho das matrizes, mas também o tempo de desenvolvimento. A GM já completou 30 projetos usando o "Processo de QFD Simplificado”, de 1994 a 1999 (Ross, 1999).

Segundo Bier e Cornesky (2001), a Universidade RainStar no Arizona está usando o QFD para analisar criticamente o seu currículo do curso de mestrado em “Acupuntura e Medicina Oriental”, com o objetivo de atualizá-lo de modo que atenda as necessidades dos seus clientes. Neste processo é utilizada apenas uma versão modificada da Matriz da Qualidade, sem realizar análise competitiva com os currículos das outras universidades e com adaptações nos cálculos feito na matriz, para verificar se os requisitos dos clientes já são atendidos pelas características do currículo. Nesta matriz é feito o relacionamento entre os requisitos, que são todos os conhecimentos práticos necessárias para o profissional realizar sua profissão de modo competente, com as características da qualidade, que são todas as disciplinas do currículo atual. Segundo os autores, o QFD é uma importante ferramenta para analisar ou construir currículos. Com o uso do QFD foi possível identificar os pontos chaves que o curso deve focar para atender estas necessidades da profissão.

A empresa Ph.D informática utilizou o método do QFD para desenvolver um software de custos (Sonda et al., 2000). O modelo conceitual era formado por sete matrizes. Segundo a empresa, com o uso do QFD, ela verificou que tinha uma visão equivocada do que era importante para os seus clientes no software. O uso deste método possibilitou corrigir esta visão, além de controlar o processo de desenvolvimento e manutenção do software. O método também possibilitou identificar os processos críticos e democratizar e homogeneizar o conhecimento entre os membros da equipe.

\subsection{Histórico dessa pesquisa}

A pesquisa realizada no Brasil se iniciou em 1999, quando foi realizado um estudo tipo survey com uma amostra de 111 elementos. Os objetivos do estudo eram verificar empresas que utilizavam ou tinham planos de utilizar o QFD, os motivos que as levaram a usar o método e quais as dificuldades e benefícios da implantação. Os resultados desta pesquisa foram apresentados por Miguel e Carpinetti (1999). Ainda sobre os resultados do estudo de 1999 foram publicados mais dois trabalhos. O primeiro por Carnevalli, 
Sassi e Miguel (2001a) onde foram apresentados apenas os resultados do uso do QFD no setor automobilístico, e o segundo por Carnevalli e Miguel (2002). Esse último apresenta uma comparação do estudo realizado em 1999 no Brasil com as pesquisas realizadas na Suécia (Ekdahl e Gustafsson, 1997), Reino Unido (Martins e Aspinwall, 2001), nos EUA e no Japão (Cristiano et al., 2000).

No ano de 2000 foi realizado um segundo estudo, mais aprofundado sobre o uso do QFD no Brasil, com uma amostra de 506 elementos, e com os mesmos objetivos do estudo de 1999. A metodologia de pesquisa desse segundo estudo pode ser encontrado em Carnevalli e Miguel (2001), sendo que os resultados dos questionários pilotos foram apresentados por Carnevalli, Sassi e Miguel (2001b).

No presente trabalho serão apresentados os resultados do estudo concluído em 2002, comparando-o com a pesquisa realizada no Reino Unido (Martins e Aspinwall, 2001). É importante ressaltar que o artigo de Carnevalli e Miguel (2002) é diferente do presente trabalho, pois no artigo de 2002 (Carnevalli e Miguel 2002) são comparados os resultados da pesquisa sobre o uso do QFD em algumas empresas realizada em 1999 (Miguel e Carpinetti, 1999) e no presente texto são comparados os resultados concluídos em 2002.

\section{Análise da comparação entre os dois estudos}

Será apresentada, a seguir, a comparação dos resultados da pesquisa realizada no Reino Unido com os resultados parciais da pesquisa Brasileira. Com relação ao objetivo de cada pesquisa pode-se citar:

- Pesquisa Brasileira: verificar o grau de introdução do QFD no Brasil, estudando principalmente as maiores empresas privadas, por faturamento, identificando quais são os benefícios e dificuldades de implantação do QFD. Também identificar empresas que já tinham maturidade no uso do método.

- Pesquisa do Reino Unido: identificar benefícios e dificuldades de implementação no país, e quais são os pontos chaves de implantação.

Como pode ser notado, as duas pesquisas têm objetivos muito semelhantes. Em relação ao tipo de amostra e técnica de coleta de dados as duas pesquisas utilizam metodologias muito parecidas, com amostra não aleatória e intencional e o uso do questionário via correio como instrumento de coleta de dados, existindo diferenças apenas no tamanho e seleção da amostra. As duas pesquisas também realizaram pré-testes para aperfeiçoar o instrumento de coleta de dados

Na pesquisa Brasileira foi utilizada uma amostra de 506 elementos composta da seguinte maneira: empresas que estavam na classificação da revista Exame, entre as 500 maiores empresas privadas, por vendas (Exame, 2000), e empresas que não estavam nesta classificação, mas que a equipe tinha conhecimento de estarem usando, implementado, ou tinham planos de implementar o QFD, conforme o estudo preliminar anterior realizado por Miguel e Carpinetti (1999).

A pesquisa do Reino Unido (Martins e Aapinwall, 2001), utilizou uma amostra de 246 elementos. A amostra era composta da seguinte maneira: 100 principais empresas do Reino Unido por vendas, mais 120 empresas do setor automotivo que tinham ISO 9000 ou equivalente, das quais foram selecionadas 211 empresas. Além destas empresas faziam parte da amostra, 24 principais universidades (segundo o jornal The Times), e foi contactada uma empresa que indicou o nome de outras 11 empresas em Midlands que poderiam estar usando o QFD, completando assim os 246 elementos.

Na pesquisa do Reino Unido, o questionário piloto foi enviado para duas empresas, uma que não usava o QFD e outra que poderia estar usando o método. Já a pesquisa brasileira realizou dois pré-testes, sendo que o primeiro foi o envio do questionário para dois pesquisadores de outras universidades que estudam o QFD, buscando-se uma análise crítica do instrumento. O segundo pré-teste foi semelhante ao da pesquisa do Reino Unido, onde o questionário piloto foi enviado para três empresas da amostra, uma que não usava o QFD, outra que estava implementado o método e uma que já usava o QFD, há algum tempo.

A Tabela 1 apresenta os índices de resposta das pesquisas. Verifica-se que o Reino Unido teve um índice de quase $28 \%$, enquanto a pesquisa Brasileira teve um índice de quase $21 \%$ dos casos. Apesar disso, nos dois casos esses índices estão dentro do recomendado para questionários via correio, que pode ser de $3 \%$ a 50\% (Mattar, 1996) ou entre 20 a $40 \%$ (Furtrell, 1994).

Tabela 1 - Resultados das amostras

\begin{tabular}{|c|c|c|}
\hline Pesquisa & Amostra & Índice de Retorno \\
\hline Brasil & 506 & $20,9 \%$ \\
\hline Reino Unido & 246 & $27,9 \%$ \\
\hline
\end{tabular}


A pesquisa realizada no Reino Unido e no Brasil identificaram números próximos de empresas que estão utilizando o QFD. No Reino Unido foram identificadas 19 empresas e no Brasil 20 empresas utilizando o método.

Com os resultados apresentados na Tabela 2 pode-se verificar que o QFD é pouco utilizado no Brasil e no Reino Unido. Na pesquisa brasileira, apenas pouco mais de $18 \%$ estão utilizando o método, enquanto que no Reino Unido, apesar deste índice ser um pouco melhor, ele fica abaixo dos $30 \%$.

Tabela 2 - Porcentagem em relação ao índice dos que usam ou não o QFD

\begin{tabular}{|c|c|c|}
\hline Pesquisa & $\begin{array}{c}\text { Empresas que } \\
\text { usam o QFD }\end{array}$ & $\begin{array}{c}\text { Empresas que não } \\
\text { usam o QFD }\end{array}$ \\
\hline Brasil & $18,9 \% *$ & $81,1 \% *$ \\
\hline Reino Unido & $27,5 \%$ & $72,5 \%$ \\
\hline
\end{tabular}

*Desconsiderando os questionários recebidos em branco

A Figura 1 apresenta a classificação das empresas que utilizam o QFD nos dois países em setores de manufatura, serviços e educação. Verifica-se que o setor de manufatura é o mais destacado, sendo que no Brasil o índice deste setor é de 90\% e no Reino Unido supera $73 \%$ dos casos. O uso do QFD principalmente no setor de manufatura era esperado, pois o método foi criado inicialmente para desenvolvimento de produtos. Entretanto, tem-se verificado também numa pesquisa americana (Cristiano et al., 2000), realizada simultaneamente nos EUA e no Japão, um amplo uso também no setor de serviços.

O uso do QFD no setor de serviços é mais que o dobro no Reino Unido do que no Brasil. Não estão claros quais são os motivos, mas uma futura pesquisa poderia verificar se este fato está relacionado com o nível de divulgação do QFD em cada país. Verificouse na pesquisa do Reino Unido que 5\% das empresas estão utilizando o QFD são do setor de educação. No Brasil este índice ficou em $0 \%$ pois o setor de educação não foi investigado pela pesquisa, não fazendo parte de sua amostragem.

A Figura 2 apresenta o porte das empresas que utilizam o QFD, de acordo com o número de funcionários. Para isto considerou-se como empresas de grande porte aquelas que tem mais de 300 funcionários, empresas médias com 25 a 300 funcionários e empresas pequenas, menos de 25 funcionários. Essa classificação foi feita de forma que compatibilizasse ambas as pesquisas, uma vez que essas faixas foram adotadas pela pesquisa feita no Reino Unido.

Verifica-se, no Brasil, que quase a totalidade das empresas que utilizam o QFD são de grande porte (90\% dos casos). Somente em mais de 5\% dos casos o método é utilizado em empresas de médio porte. Entretanto, deve-se considerar que a amostra desta pesquisa é composta principalmente pelas 500 maiores empresas privadas por vendas, tendo em vista que poucas empresas de médio e pequeno porte fazem parte da amostra. Mais de 63\% dos casos, no Reino Unido, são empresas de grande porte que utilizam o QFD e em 36\% empresas de médio porte. Também no caso da pesquisa do Reino Unido, o tipo de amostra incluía as 100 principais empresas por vendas, o que pode ter influenciado os resultados. Em nenhuma das duas pesquisas foram encontradas empresas com menos de 25 funcionários usando o QFD. Entretanto, apesar disso, não se deve considerar que o método do QFD só deva ser usado por empresas grandes ou de médio porte, pois Paiva e Cheng (2001) já demonstraram que é possível implementar o QFD numa empresa com limitações de recursos e inexistência de planejamento estratégico. Apesar de seus estudos serem feitos numa empresa com 60 funcionários, a falta de recursos apresentados são características encontradas nas empresas de pequeno porte.

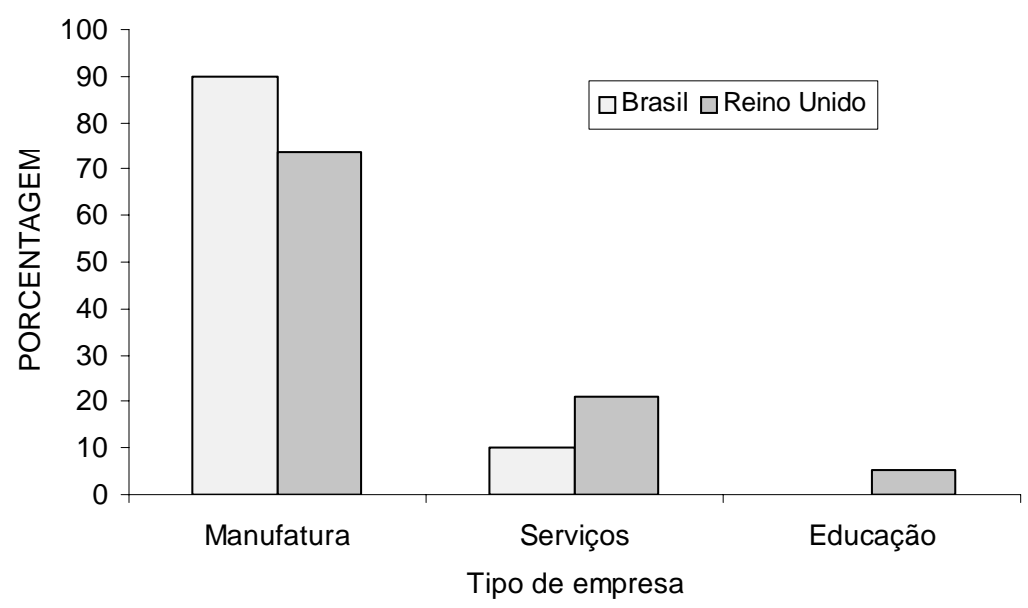

Figura 1 - Setor de atuação 
A Figura 3 apresenta os principais motivos que levaram as empresas dos dois países a iniciarem a implantação do QFD. No Brasil, as empresas iniciaram a implantação buscando melhoria do processo de desenvolvimento de produtos (em $85 \%$ dos casos), a busca dos benefícios do método citada na literatura e aumento da satisfação dos clientes (cada um com 80\% dos casos). Na literatura (Cheng et al., 1995; Ohfuji et al., 1997), alguns benefícios citados do uso do QFD são: redução no número de modificações de projetos; aumento da satisfação dos clientes; diminuição do número de reclamações; diminuição do tempo de desenvolvimento do produto a partir da segunda aplicação; redução de custos; melhoria da comunicação entre departamentos.

No Reino Unido, se destacaram os motivos de redução das reclamações dos clientes (em mais de $33 \%$ dos casos) e atendimento dos objetivos da filosofia do TQM (em 24\% dos casos).
Sobre se as empresas contrataram uma consultoria para ajudar na implantação do QFD, verificou-se que a maioria não contratou. No Brasil, apenas 30\% contratou uma consultoria e no Reino Unido, apenas $26 \%$ dos casos. Um fato interessante é que apesar da maioria das empresas não terem contratado uma consultoria na implantação, nos dois países, algumas delas tiveram dificuldades por falta de experiência em QFD, o que eventualmente poderia ser resolvido com o apoio de uma consultoria.

Em relação ao tempo de experiência no uso do QFD, mais de 94\% das empresas do Reino Unido tem pelo menos três anos. Na pesquisa brasileira, o número de empresas que tem pelo menos três anos de experiência em QFD cai para mais de 65\% dos casos, indicando que o seu uso é um pouco mais recente no Brasil.

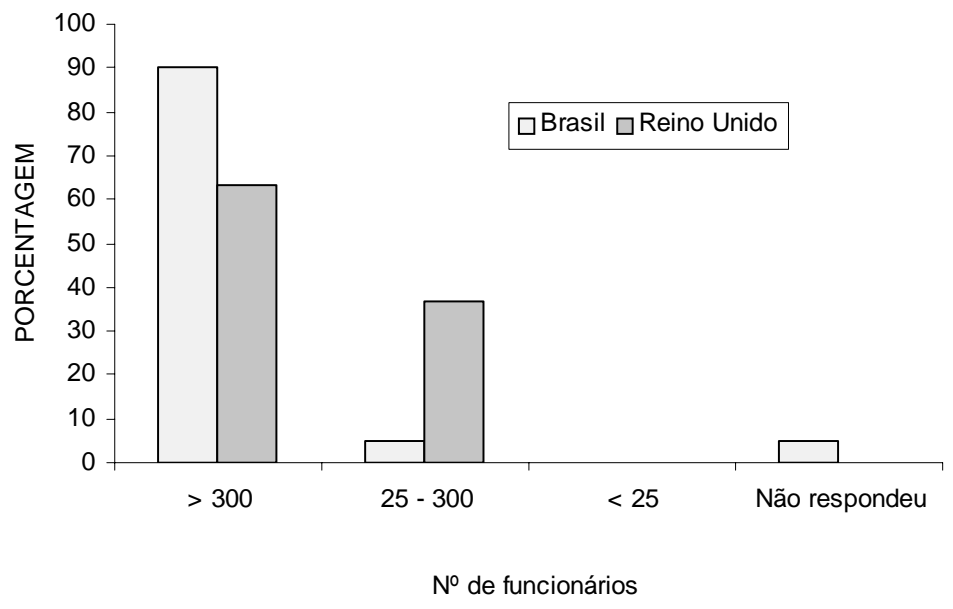

Figura 2 - Número de funcionários das empresas

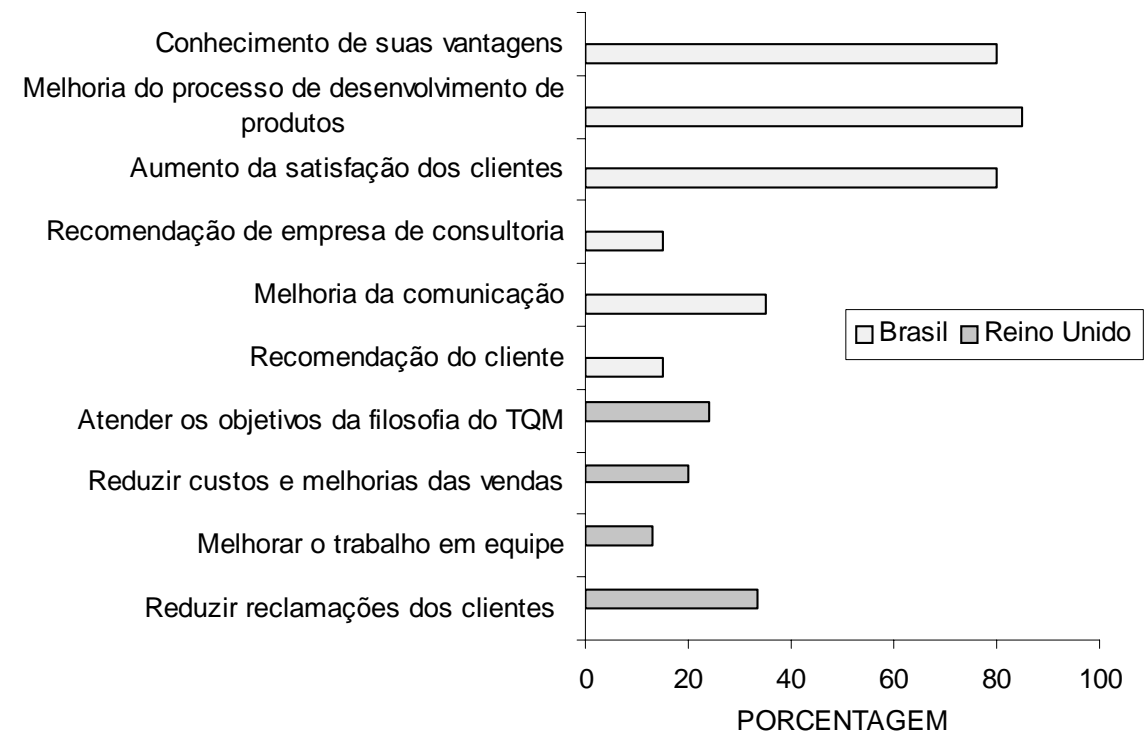

Figura 3 - Motivos da implantação do QFD*

*Os dados da pesquisa Brasileira são a somatória das alternativas “concordo” com “concordo totalmente” 
Tabela 3 - Dificuldades na implantação do QFD

\begin{tabular}{|l|c|c|}
\hline Dificuldades & Brasil* & Reino Unido \\
\hline Conflitos de opiniões nos grupos & $25,0 \%$ & $0,0 \%$ \\
\hline A(s) matriz(es) não ajudou(ram) na sistematização & $5,0 \%$ & $0,0 \%$ \\
\hline Falta de treinamento & $20,0 \%$ & $0,0 \%$ \\
\hline Dificuldade de interpretar os requisitos dos clientes & $35,0 \%$ & $9,5 \%$ \\
\hline A(s) matriz(es) é(são) muito grande(s) & $45,0 \%$ & $0,0 \%$ \\
\hline Dificuldade em atribuir peso aos requisitos dos clientes & $30,0 \%$ & $0,0 \%$ \\
\hline Falta de tempo para conduzir a consulta aos clientes & $25,0 \%$ & $0,0 \%$ \\
\hline Falta de experiência em QFD & $50,0 \%$ & $16,7 \%$ \\
\hline Falta de recursos & $35,0 \%$ & $7,1 \%$ \\
\hline Comprometimento dos membros do grupo & $50,0 \%$ & $14,3 \%$ \\
\hline Falta de suporte gerencial & $40,0 \%$ & $14,3 \%$ \\
\hline Dificuldade em função da estrutura organizacional da empresa & $35,0 \%$ & $0,0 \%$ \\
\hline Tempo consumido & $0,0 \%$ & $16,7 \%$ \\
\hline Completar as Matrizes & $0,0 \%$ & $7,1 \%$ \\
\hline Dificuldade em comparar com os concorrentes & $0,0 \%$ & $4,8 \%$ \\
\hline Trabalho em equipe & $0,0 \%$ & $4,8 \%$ \\
\hline Não alcançou os resultados & $0,0 \%$ & $2,4 \%$ \\
\hline
\end{tabular}

* Os dados da pesquisa Brasileira apresentados são a somatória das alternativas "concordo" com "concordo totalmente"

A Tabela 3, apresenta as principais dificuldades encontradas nas duas pesquisas. No Brasil, se destacaram as dificuldades: "falta de experiência em QFD”; "comprometimento dos membros do grupo"; "a(s) matriz(es) é (são) muito grande(s)”; "falta de suporte gerencial”. No Reino Unido, as principais dificuldades também são “falta de experiência em QFD”; “comprometimento dos membros do grupo"; "falta de suporte gerencial”, também se destacando como problema o tempo consumido no uso do método.

Segundo Cheng et al. (1995) e Ohfuji, et al. (1997), o apoio da alta gerência é muito importante para que a implantação do QFD tenha sucesso. Também segundo Cheng et al. (1995), a escolha adequada da equipe com um líder que tenha autoridade sobre o desempenho do grupo é um fator de sucesso, evitando a falta de comprometimento. Segundo os autores, as equipes de QFD onde os líderes não tem nenhuma autoridade ou poder de avaliação dos seus membros, dificilmente tem sucesso, isto porque cada elemento da equipe costuma dar prioridade ao seu trabalho do dia a dia, em prejuízo do projeto do QFD.

Sobre como são obtidos os requisitos dos clientes, verificou-se que no Brasil utiliza-se em 50\% dos casos pesquisas diretas e indiretas com os clientes. Em 20\% dos casos realizam somente pesquisas diretas e outros 20\% somente pesquisas indiretas (10\% das empresas não responderam esta questão). No Reino Unido, 58\% realizam apenas pesquisas diretas e 15\% dos casos somente pesquisas indiretas. Esta pesquisa não informou se encontrou empresas que utilizassem os dois tipos de pesquisas.
Para Ohfuji et al. (1997) é muito importante realizar pesquisas diretas com os clientes, para que as empresas possam descobrir quais seus verdadeiros requisitos. Deste modo, a maioria das empresas estão pesquisando a voz do cliente de forma adequada. Também é importante para a empresa analisar dados indiretos, como relatórios de reclamação e dados de garantia, assim realizando pesquisas diretas e indiretas, ao mesmo tempo a empresa consegue identificar os requisitos dos clientes e as falhas do seu produto que devem ser corrigidas.

Ao contrário do que ocorre no Brasil (70\% fazem mais de uma matriz), a maioria das empresas do Reino Unido realiza apenas a matriz da Qualidade (em 63\% dos casos). Este fato é preocupante, podendo prejudicar os resultados do método, pois o uso apenas da matriz da qualidade não garante que a empresa consiga fabricar produtos com os requisitos dos clientes definidos nesta matriz.

A Tabela 4 apresenta os principais benefícios do uso do QFD encontrados nas duas pesquisas. Na pesquisa brasileira, se destacam os benefícios mensuráveis (por exemplo: redução dos custos em 5\%) e os não mensuráveis (por exemplo: melhoria do trabalho em grupo e da comunicação entre os departamentos). Na pesquisa do Reino Unido, também aparecem os dois tipos de benefícios, mas diferentemente da pesquisa brasileira se destacam os benefícios não mensuráveis (por exemplo: aumento da participação dos empregados no processo de decisões). Esta diferença de resultados de um país para o outro, pode ter alguma relação com o fato da maioria das empresas do Reino 
Tabela 4 - Benefícios encontrados com o uso do QFD

\begin{tabular}{|l|c|c|}
\hline \multicolumn{1}{|c|}{ Benefícios } & Brasil & Reino Unido \\
\hline Aumento da qualidade e confiabilidade & $45 \%$ & $39 \% \mathrm{~A}$ \\
\hline Aumento da satisfação do cliente & $50 \%$ & - \\
\hline Aumento da satisfação dos funcionários & $25 \%$ & - \\
\hline Melhoria do trabalho em grupo & $50 \%$ & $20 \%$ \\
\hline Fortalecimento das práticas de engenharia Simultânea & $40 \%$ & - \\
\hline Redução de custos & $30 \%$ & $39 \% \mathrm{~A}$ \\
\hline Melhoria da comunicação entre departamentos & $50 \%$ & $15 \% \mathrm{~B}$ \\
\hline Redução no tempo de lançamento de novos produtos & $25 \%$ & $39 \% \mathrm{~A}$ \\
\hline Redução no número de alterações após lançamento & $30 \%$ & - \\
\hline Fornecer uma melhor visão sobre os concorrentes & $35 \%$ & - \\
\hline Melhoria da comunicação entre o mercado e o projeto & - & $15 \% \mathrm{~B}$ \\
\hline Redução no número de reclamações & - & $39 \% \mathrm{~A}$ \\
\hline Aumento da participação dos empregados no processo de decisões & - & $24 \%$ \\
\hline Outros & $10 \%$ & - \\
\hline $\begin{array}{l}\text { Nota: Os dados da pesquisa Brasileira apresentados são a somatórias das alternativas "bom" com "excelente". Os dados da } \\
\text { pesquisa do Reino Unido significam A: a somatórias das respostas aumento da qualidade e confiabilidade, mais redução de } \\
\text { custos e no tempo de lançamento de novos produtos, mais aumento da lealdade aos requisitos, mais redução no número de } \\
\text { reclamações = 39\%; e em B: melhoria da comunicação entre departamentos e entre o mercado e o projeto = 15\%.) }\end{array}$ \\
\hline
\end{tabular}

Unido realizarem apenas a matriz da qualidade, mas para confirmar ou não esta hipótese seria necessário realizar um estudo mais aprofundado em cada país.

Sobre a divulgação dos resultados do QFD, verificouse na pesquisa do Reino Unido que, em $13 \%$ dos casos, a empresa os divulgou para clientes e fornecedores, mas, em 50\%, os resultados são considerados sigilosos e só são divulgados internamente, conforme a necessidade do setor. Na pesquisa brasileira, os resultados também são semelhantes. Em 10\% dos casos são divulgados externamente, em 25\% dos casos os resultados são divulgados internamente conforme as necessidades básicas e em 35\% apenas para o pessoal chave. Em 15\% consideram as informações como confidenciais, ficando com a equipe do QFD. Na verdade, seria muito importante que os resultados do QFD fossem divulgados para todos os setores da empresa, pois, desta forma, se conscientizaria os funcionários sobre quais são os requisitos dos clientes e de como o seu trabalho interfere na qualidade final do produto. Além disso, a divulgação democratizaria o conhecimento, não ficando concentrado somente em algumas pessoas da empresa.

Um dado preocupante é que 58\% dos casos estudados no Reino Unido não fazem avaliação da implantação do QFD. No Brasil, este número também é alto, ficando em 45\% dos casos. Sem a avaliação da implantação não é possível verificar se os benefícios da aplicação do método compensaram o investimento feito pela empresa.

No Brasil, 25\% das empresas que utilizavam o QFD interromperam o seu uso devido aos seguintes problemas: as expectativas não foram alcançadas (em $60 \%$ dos casos); surgiram problemas para implementa-lo (em 40\% dos casos); decisão gerencial (em 20\% dos casos), e outros fatores (em $40 \%$ dos casos) tais como dificuldade devido a estrutura da engenharia. Essas empresas também citaram problemas como falta de adaptação às características da empresa, treinamento insuficiente, processo de implementação do QFD deficiente e não aplicado adequadamente, utilização do QFD é complexa, aplicação depende da experiência do facilitador da ferramenta e dependendo do tipo de projeto ela se torna muito trabalhosa para ser utilizada.

No Reino Unido, quase 37\% das empresas (7 empresas) também abandonaram o uso do método. Destas, 6 empresas (quase 32\%) tiveram problemas na implantação, como falta de comprometimento da equipe do QFD.

Serão apresentadas a seguir as conclusões de cada pesquisa.

\section{Conclusões}

A pesquisa realizada no Reino Unido verificou que as dificuldades do uso do QFD são de trabalhar em equipes, tempo gasto, custo e a complexidade do método. Além disso, as empresas tem dificuldade em listar e definir a voz do cliente, e a maioria só faz a primeira matriz do QFD. Os benefícios encontrados: são melhoria do trabalho em equipe, na comunicação com os clientes internos e externos e maior envolvimento dos funcionários. 
Por sua vez, a pesquisa brasileira verificou que o QFD ainda é pouco utilizado no Brasil se concentrando no setor de manufatura e sua utilização é feita principalmente por empresas de grande porte. O principal motivo para o início da implantação é a busca pela melhoria do processo de desenvolvimento de produto. As principais dificuldades estão relacionadas com a falta de experiência e comprometimento dos membros do grupo e dificuldades de se trabalhar com matrizes grandes. Até o momento, foram identificadas cinco empresas com experiência em QFD. Essas empresas têm mais de três projetos concluídos, e usa o método há, pelo menos, dois anos.

Para fins comparativos, em primeiro lugar é importante ressaltar que as pesquisas foram realizadas em anos próximos, mas diferentes. Deste modo, estas comparações só são válidas para a realidade dos países nos anos da realização de cada pesquisa.

Com a comparação das duas pesquisas, verificou-se que os objetivos eram muito semelhantes, as duas buscavam identificar os benefícios e dificuldades de se implementar o QFD. Para atingir estes objetivos, as duas pesquisas utilizaram amostras não aleatórias e intencionais, usando o questionário via correio para coletar os dados das pesquisas.

A maioria das empresas dos dois países utilizam o QFD há pelo menos 3 anos. Uma das grandes diferenças na aplicação do método nos dois países é que, enquanto no Brasil a maioria das empresas realiza mais de uma matriz, no Reino Unido mais da metade só faz a casa da qualidade. Em relação às dificuldades de implantação, destacam-se nos dois países problemas por falta de experiência em QFD e falta de comprometimento dos membros do grupo. Os principais benefícios encontrados com o uso do QFD variam com as pesquisas. Enquanto no Reino Unido se destacaram os benefícios não mensuráveis, no Brasil tem se encontrado os dois tipos de benefícios. Um fato preocupante nas duas pesquisas é que na maioria dos casos não se faz avaliação da implantação do QFD. Deste modo, fica difícil demonstra claramente a viabilidade da implantação do método. A tabela 4 apresentada anteriormente, mostra os benefícios do método indicando que algum tipo de avaliação, mesmo que qualitativa é realizada. Porém, estudos mais aprofundados sob esses aspectos devem ser realizados. Essa é a próxima etapa desse trabalho.

\section{Referências}

AKAO, Y. Introdução ao desdobramento da qualidade. Belo Horizonte: Fundação Christiano Ottoni, 1996. 187 p.
BIER, I.D.; CORNESKY,R. Using QFD to construct a higher education curriculum: Quality tool ensures meeting of customer and accreditation needs, Quality Progress, Milwaukee, v.34, n.4, p.64-68, Apr. 2001.

CARNEVALLI, J. A.; MIGUEL, P. A. C. Desenvolvimento da pesquisa de campo, amostra e questionário para realização de um estudo tipo survey sobre a aplicação do QFD no Brasil. In: ENCONTRO NACIONAL DE ENGENHARIA DE PRODUÇÃO, 16., Salvador, 2001. Anais...Porto Alegre: SONOPRESS ${ }^{\circ}$, 2001. 1 CD.

CARNEVALLI, J. A.; MIGUEL, P. A. C. QFD application in different counties: a comparison between an exploratoy study in Brazil with other surveys. Revista Product: Management \& Development Revista Brasileira de Gestão de Desenvolvimento de Produto. Florianópolis, v.1, n.2, p. 5-18, 2002.

CARNEVALLI, J. A.; SASSI, A.; MIGUEL, P. A. C. Análise da aplicação do desdobramento da função qualidade (QFD) em empresas do segmento automotivo. In: SIMEA 2001 - SIMPÓSIO INTERNACIONAL DE ENGENHARIA AUTOMOTIVA, 11., São Paulo, 2001. Anais...São Paulo, 2001a. 1 CD.

CARNEVALLI, J. A.; SASSI, A.; MIGUEL, P. A. C. Implantação do QFD no Brasil: base metodológica e resultados do piloto. In: CONGRESSO BRASILEIRO DE GESTÃO DE DESENVOLVIMENTO DE PRODUTO, 3., Florianópolis, 2001.

Anais...Florianópolis, 2001b. 1 CD.

CHENG, L. C., et al. QFD: planejamento da qualidade. Belo Horizonte: Fundação Christiano Ottoni, 1995. 261p.

CRISTIANO et al. Customer-driven product development through quality function deployment in the U.S. and Japan. J Prod Innov Manag, New York: Elsevier Science, n.17, p.286-308, 2000.

EKDAHL, F.; GUSTAFSSON, A. QFD: the Swedish experience. In: THE NINTH SYMPOSIUM ON QUALITY FUNCTION DEPLOYMENT, 9., Novi, 1997. Anais... Novi: Michigan, 1997, p.15-27.

EXAME, Melhores e maiores 2000. São Paulo: Editora Abril, Jun. de 2000. Edição especial.

FURTRELL, D. Ten reasons why surveys fail. Quality Progress, p.65-69, Apr. 1994. 
MARTINS, A. \& ASPINWALL, E. M. Quality function deployment: an empirical study in the UK. Total Quality Management, v.12, n.5, p.575-588, 2001.

MATTAR, F.N. Pesquisa de marketing: edição compacta. São Paulo: Atlas, 1996. 270 p.

MIGUEL, P.A.C.; CARPINETTI, L.R. Some Brazilian experiences on QFD application. In: INTERNATIONAL SYMPOSIUM ON QUALITY FUNCTION DEPLOYMENT, 5., Belo Horizonte, 1999. Anais... Belo Horizonte: UFMG, 1999. p. 229239.

PAIVA, C.L.; CHENG, L.C. O emprego do QFD como ferramenta para a implantação do processo de desenvolvimento de novos produtos em uma pequena empresa de massas alimentícia. In: CONGRESSO BRASILEIRO DE GESTÃO DE DESENVOLVIMENTO DE PRODUTO, 3., Florianópolis, 2001. Anais... Florianópolis, 2001. 1CD.

OHFUJI, T.; ONO, M. \& AKAO, Y. Métodos de desdobramento da qualidade (1). Belo Horizonte: Fundação Christiano Ottoni, 1997. 256 p.

ROSS, H.QFD status at General Motors in the U.S. In: INTERNATIONAL SYMPOSIUM ON QUALITY FUNCTION DEPLOYMENT, 5, Belo Horizonte, 1999. Anais... Belo Horizonte: UFMG, 1999.

SONDA, F.A.; RIBEIRO, J.L.D.; ECHEVESTE. A aplicação do QFD no desenvolvimento de software: um estudo de caso, Produção, São Paulo, v. 10 n.1, p.51-75, Dez, 2000.

\section{Agradecimentos}

Os autores agradecem as seguintes instituições e pessoas, sem as quais não seria possível a realização desse trabalho: FAPESP (bolsa de mestrado, processo: 00/06220-2), CNPq (bolsa de produtividade em pesquisa, processo 301202/97-4 e bolsa de iniciação científica do Programa Institucional da UNIMEPCNPq), à Andreza Sassi aluna de iniciação científica do programa PIBIC - CNPq, pelo auxílio e dedicação durante o andamento deste trabalho e ao Dr. Rob Hunt da Macquarie University, Austrália, e à Sra. Elaine Aspinwall da Universidade de Birmingham, Inglaterra, por cederem seus questionários de pesquisa e discussão acerca de seus resultados. Também agradecem ao Prof. Dr. Luiz César R. Carpinetti da EESC-USP pela colaboração na revisão da primeira versão completa do questionário (antes do envio do piloto para as empresas) e ao Srs. Gyorgy Henyei
Júnior e Márcio A. Querichelli pela contribuição na revisão do piloto do questionário, ao Prof. Dr. Alvaro J. Abackerli por ter cedido os arquivos de pesquisa similar realizada, e também às empresas que participaram da pesquisa e devolveram o questionário. 